\title{
Sorafenib sensitizes melanoma cells to vemurafenib through ferroptosis
}

\author{
Fengjie Tang ${ }^{1}$, Shiyan $\mathrm{Li}^{2}$, Daisong Liu ${ }^{1}$, Jian Chen ${ }^{3}$, Chaofei Han ${ }^{1}$ \\ ${ }^{1}$ Department of Burn and Plastic Surgery, The Third Xiangya Hospital of Central South University, Changsha 410013 China; ${ }^{2}$ Department of \\ Burns and Plastic Surgery, Nanjing Drum Tower Hospital, the Affiliated Hospital of Nanjing University Medical School, Nanjing 210008 China; \\ ${ }^{3}$ Department of Burns and Plastic Surgery, The First Hospital of Putian City, Putian 351100, China \\ Contributions: (I) Conception and design: F Tang, C Han; (II) Administrative support: C Han; (III) Provision of study materials or patients: S Li; (IV) \\ Collection and assembly of data: D Liu; (V) Data analysis and interpretation: J Chen; (VI) Manuscript writing: All authors; (VII) Final approval of \\ manuscript: All authors. \\ Correspondence to: Chaofei Han. Department of Burn and Plastic Surgery, The Third Xiangya Hospital of Central South University, Tongzipo Road \\ 138, Changsha 410013, China. Email: 491616@163.com.
}

Background: $B R A F$ gene mutation causes melanoma patients to develop drug resistance after 8-9 months BRAF inhibitors treatment. Therefore, overcoming BRAF inhibitor resistance has important implications for improving patient survival. Sorafenib directly inhibits tumor cell proliferation by blocking the RAF/MEK/ERKmediated cell signaling pathway. It remains unknown that whether the combination of sorafenib with vemurafenib could sensitize melanoma cells to vemurafenib, and the underlying mechanism needs to be clarified.

Methods: Vemurafenib resistant melanoma cells A375/Vem and SK-Mel-28/Vem were established by exposing to a series of concentration of vemurafenib. Cell viability was measured when A375 and SK-Mel-28 cells treated with vemurafenib or combined with sorafenib. Meanwhile the levels of Iron, GSH, MDA and reactive oxygen species (ROS) were detected. Finally we examined that whether sorafenib sensitizes melanoma cells to vemurafenib through ferroptosis.

Results: We found that sorafenib sensitized melanoma cells to vemurafenib. Sorafenib treatment did not significantly alter the production of ROS and the content of iron, GSH and MDA in vemurafenib resistant cells, but cotreatment of sorafenib and vemurafenib dramatically upregulated ROS production, MDA and iron, but decreased GSH concentration. Interestingly, sorafenib strongly promoted vemurafenibinduced cell death, which was blocked by lipid peroxidation inhibitors ferrostatin-1 but not ZVAD-FMK or necrosulfonamid.

Conclusions: Sorafenib sensitized melanoma cells to vemurafenib by increasing ROS production through ferroptosis. Our study reveals that the combination of sorafenib may provide a novel strategy of vemurafenib resistant melanoma therapy.

Keywords: BRAF inhibitors; sorafenib; ferroptosis; reactive oxygen species (ROS); resistance

Submitted Aug 14, 2019. Accepted for publication Jan 16, 2020.

doi: $10.21037 /$ tcr.2020.01.62

View this article at: http://dx.doi.org/10.21037/tcr.2020.01.62

\section{Introduction}

Melanoma (MM) is a kind of malignant tumor which originates from melanocytes, and accounts for $3-5 \%$ of skin malignant tumors. It has high malignancy and is prone to invasion and metastasis (1), resulting high mortality rate (2). BRAF kinase is the most important subtype of the Raf family in the MAPK signaling pathway (3). BRAF overexpression or mutation is closely related to the occurrence of melanoma (4). More than $60 \%$ melanomas have $\mathrm{BRAF}^{\mathrm{V} 600}$ mutation, which may encodes a constitutively 
activated BRAF protein and induces the aberrant mitogenactivated protein kinase (MAPK) signaling (5). Currently, the BRAF inhibitor vemurafenib is approved for melanoma treatment, which inhibits the kinase activity mainly by competitively binding to the ATP binding site (6). Although BRAF inhibitor has a rapid efficacy and good response rate, most melanoma patients develop tumor progression and resistance after 8-9 months of treatents resulting from reactivation of the MAPK pathway and other signaling pathways (7), It's seriously affects the survival, prognosis of patients. Therefore, overcoming BRAF inhibitor resistance has important implications for improving patient survival.

Ferroptosis is a recently recognized form of regulated cell death driven by small molecules or conditions that induce lipid-based reactive oxygen species (ROS) accumulation, and this iron-dependent form of cell death is morphologically and genetically distinct from apoptosis, necroptosis, and autophagy $(8,9)$. Ferroptosis is characterized by cell volume shrinkage and increased mitochondrial membranes and is mediated by iron-dependent lipid peroxide accumulation (10). The ferroptosis-inducing compounds, such as erastin and Ras selective lethal 3 (RSL3) could inactivate cellular glutathione (GSH)-dependent antioxidant defenses, leading to the accumulation of toxic lipid ROS $(11,12)$. Glutathione peroxidase 4 (GPX4) is a key antioxidant enzyme that is responsible for removing lipid hydroperoxides within biological membranes (8). Once GPX4 inactivation, GSH will loses ability in removing the local peroxidase reaction, which eventually lead to a lipid ROS accumulation and ferroptosis.

Sorafenib is a novel multi-target anti-tumor drug, which acts on both tumor cells and tumor blood vessels (13). Sorafenib directly inhibits tumor cell proliferation by blocking the RAF/MEK/ERK-mediated cell signaling pathway, and also inhibits VEGF and platelet-derived growth factor (PDGF) receptors to block the formation of tumor neovascularization, indirectly inhibiting the growth of tumor cells (14). In addition, sorafenib-mediated cell death was associated with accumulated ROS (15). Sorafenib combination with triterpenoid oleanolic acid has highly synergistic effects on cell death and suppresses long-term clonogenic survival (16). Sorafenib stimulates significant intracellular ROS production, leading to ferroptosis in hepatocellular carcinoma (17). In melanoma, Tsoi et al. proposed that ferroptosis-inducing drugs present an orthogonal therapeutic approach to enhance the efficacy of targeted and immune therapies (18). Notably, It's reported that there is a synergistic effects of vemurafenib and sorafenib in vemurafenib-resistant A375 cells (19). Thus, we hypothesized that the combination of sorafenib with vemurafenib could sensitize melanoma cells to vemurafenib via ferroptosis.

In this study, we aim to investigate whether sorafenib could sensitize melanoma cells to vemurafenib. We found that sorafenib sensitized melanoma cells to vemurafenib by increasing ROS production through ferroptosis. Our study reveals that sorafenib may be a novel strategy for overcoming vemurafenib resistance in melanoma.

\section{Methods}

\section{Reagents}

Vemurafenib (cat No. S1267), sorafenib (cat No. S1040), ferroptosis inhibitor Ferrostatin-1 (Fer-1) (cat no. S7243), caspase inhibitor Z-VAD-FMK (cat No. S7023) and MLKL inhibitor Necrosulfonamide (cat No. S8251) were purchased from Selleck (Shanghai, China).

\section{Cell culture and treatment}

Two melanoma cell lines, A375 and SK-Mel-28, were purchased from National Infrastructure of Cell Line Resource (Beijing, China). All cells were cultured in RP1640 medium supplemented with $10 \%$ fetal bovine serum under $5 \% \mathrm{CO}_{2}$ and $37^{\circ} \mathrm{C}$ conditions for further study.

The resistant MM cells (A375/Vem and SK-Mel-28/ Vem) were established in our lab by stepwise exposure to escalatin concentrations of Vemurafenib. Briefly, A375 and SK-Mel-28 cells were treated with $0.5-6.0 \mu \mathrm{M}$ Vemurafenib every 3 days for 6 weeks. By that analogy, the final concentration reached to $6 \mu \mathrm{M}$.

For the mechanism investigation, A375 and SK-Mel-28 cells were exposed to ferroptosis inhibitor Ferrostatin-1 (Fer-1) ( $2 \mu \mathrm{M}$ dissolve in DMSO), caspase inhibitor Z-VAD-FMK (50 $\mu$ M dissolve in DMSO) and MLKL inhibitor Necrosulfonamide ( $1 \mu \mathrm{M}$ dissolve in DMSO) for 12 hours.

\section{Proliferation measurement}

Cell Counting Kit-8 (CCK-8) (Beyotime, Hangzhou, China) was used to determine cell proliferation according to the manufacturer' instructions. Briefly, five-thousand cells were seeded in each 96-well plate and treated with the indicated drugs. After that, CCK- 8 reagents $(10 \mu \mathrm{L})$ were 
added to medium. After $1 \mathrm{~h}$ incubation, a microplate reader was used to detect the OD value at $570 \mathrm{~nm}$.

\section{Biochemistry detection}

Commercial kits (cat No. MAK025, cat No. CS0260, cat No. MAK085, and cat No. MAK143 were purchased from Merck (Shanghai, China) were used to detect the iron, GSH, MDA and ROS concentrations according to the manufacturer' instructions, respectively.

\section{Western blot}

After treatment, the cells were collected and used for protein extraction using radio immunoprecipitation assay (RIPA) lysis buffer. Fifty micrograms proteins were separated by $10 \%$ SDS/PAG and transferred onto PVDF membranes. The membranes were rinsed with PBS and immunoblotted with primary antibodies (Rabbit mAb anti-NRF2 antibody, cat No. 12721, diluted at 1:1,000, from Cell Signaling Technology; mouse mAb anti-GPX4 antibody, cat No. ab16739, diluted at 1:2,000, from Abcam). Appropriate peroxidase-conjugated secondary antibody was added into the membranes and incubated for $2 \mathrm{~h}$. A BioRad (Hercules, CA) imaging system was sued to detect the specific bands.

\section{Statistical analysis}

Statistical analyses were processed by using GraphPad Prism software (GraphPad Software Inc., La Jolla, CA). Each experiment was executed at least three times, and the data are presented as means \pm SD. The difference between the two groups was processed by Student's $t$-test. The differences among multiple groups was evaluated by oneway ANOVA with Bonferroni test. Statistical significance was identified by $\mathrm{P}<0.05$.

\section{Results}

\section{The combination of vemurafenib and sorafenib inbibits melanoma cell viability}

To investigate whether sorafenib could overcome vemurafenib resistance in melanoma cells, vemurafenib resistant MM cells (A375/Vem and SK-Mel-28/Vem) were established. The morphological changes were obvious. Resistant cells were larger and flatter than their native counterparts, with abundant cytoplasm and alterations of cytoplasmic membrane shape (Figure 1A). A375/Vem and SK-Mel-28/Vem and their parental cells were exposed to a series of concentrations of vemurafenib. The cell viability was significantly decreased in all cells. However, the rates of descent in A375/Vem and SK-Mel-28/Vem cells were significantly lower than that of in their parental cells (Figure 1B), implying that A375/Vem and SK-Mel-28/ Vem cells obtain a certain degree of resistance. In addition, the IC50 of A375/Vem (4 $\mu M)$ and SK-Mel-28/Vem $(7 \mu M)$ were significantly higher than in A375 $(1 \mu \mathrm{M})$ and SK-Mel-28 (2 $\mu \mathrm{M})$ (Figure 1C). Moreover, the resistant cells maintained a highly active MAPK pathway in the presence of vemurafenib (Figure S1). Then, BRAF-wildtype cells WM35, BRAF-mutation-type cells A375, the resistant cells A375/Vem and SK-Mel-28/Vem cells, were treated with vemurafenib or combined with sorafenib and measured the cell viability. We found that vemurafenib treatment significantly inhibits $M M$ cells viability. This effect was also observed in sorafenib treated cells (Figures 2,S2). Importantly, we observed that the combination of vemurafenib with sorafenib showed a synergistic effect in inhibiting cell viability in a dosedependent manner (Figures 2,S2).

\section{The effects of sorafenib on oxidative stress in vemurafenib resistant melanoma cells}

We further investigate the mechanism of sorafenib sensitizing MM cells to vemurafenib. Ferroptosis is a new form of programmed cell death characterized with the iron-dependent lipid peroxides, the iron-dependent ROS accumulation triggers ferroptosis mainly by inactivation of GPX4 or GSH deficiency (10). So we treated the vemurafenib resistant cells with either vemurafenib or together with sorafenib $(10 \mu \mathrm{M})$ for $48 \mathrm{~h}$, then detected the level of iron and lipid oxidation related molecules. As shown in Figures 3 and 4, the concentrations of Iron, GSH, MDA (an end product of lipid peroxidation), and ROS in cells treated with vemurafenib or sorafenib alone have no significant changes compared with the control cells. However, the combination of vemurafenib and sorafenib dramatically increased the concentration of Iron, MDA, and ROS, but reduced GSH in vemurafenib resistant cells compared with the cells treated with vemurafenib and sorafenib alone. In RAF-wild-type cells WM35 and BRAF-mutation-type cells A375, the levels of Iron, GSH, MDA, and ROS were increased, but GSH were reduced 

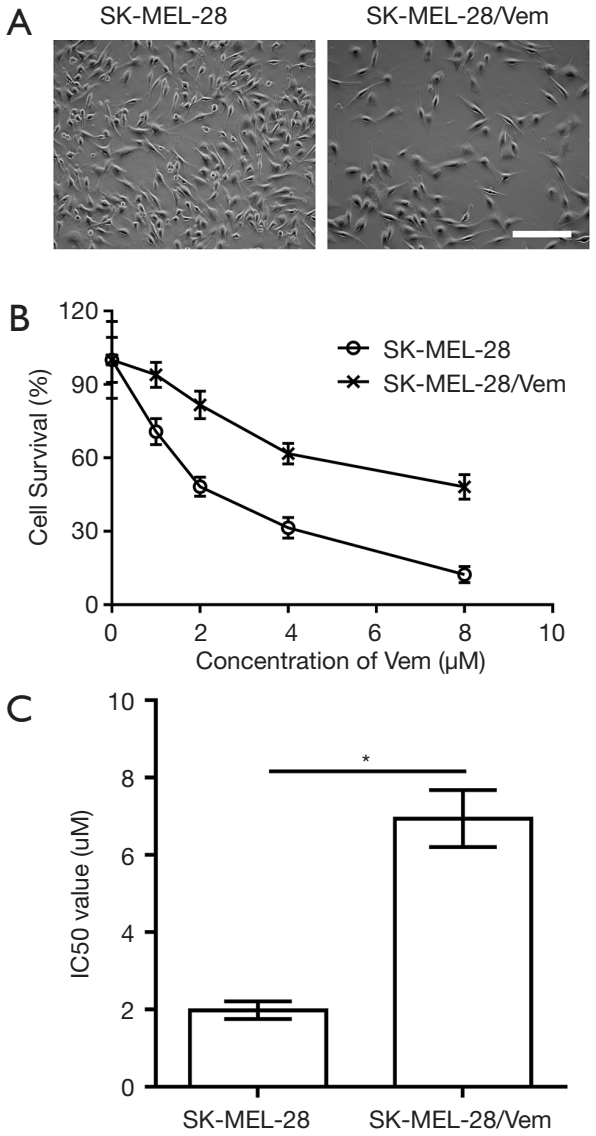
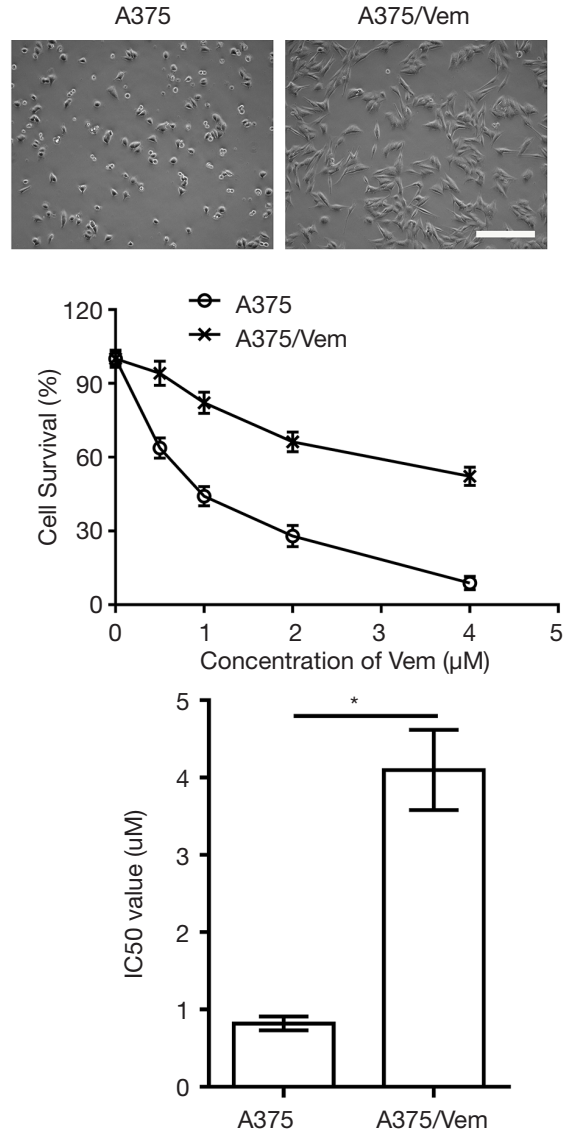

Figure 1 IC50 in vemurafenib-resistant MM cells. Vemurafenib-resistant MM cell lines were established. The cells were treated with a series of concentrations of vemurafenib (Vem). (A) The photographs obtained by light microscope showed the morphology changes of the cells. Bar $=200 \mu \mathrm{m}$. (B) CCK-8 assay was performed to measure the cell viability in vemurafenib-resistant SK-Mel-28 cells and A375 cells after 48 h-treatment. (C) IC50 was calculated for vemurafenib-resistant SK-Mel-28 cells and A375 cells and their parental cells. MM, melanoma. *, $\mathrm{P}<0.05$. and MLKL inhibitor Necrosulfonamide for 12 hours. CCK-8 assay was performed to measure the cell viability in vemurafenib-resistant A375 cells (A) and SK-Mel-28 cells (B). * $\mathrm{P}<0.05$.

A

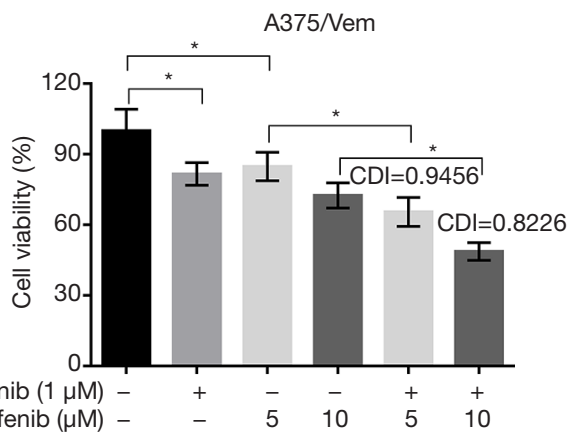

B

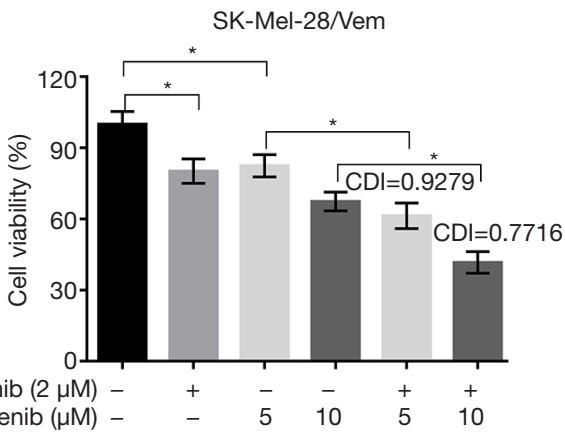

Figure 2 Sorafenib sensitizes MM cells to vemurafenib. The vemurafenib-resistant cells were treated with vemurafenib or sorafenib alone, or both of them for 48 hours. CCK-8 assay was performed to measure the cell viability in vemurafenib-resistant A375 cells (A) and SKMel-28 cells (B) after 48 h-treatment. *, $\mathrm{P}<0.05$. The coefficient of drug interaction $(\mathrm{CDI})$ was calculated as follows: $\mathrm{CDI}=\mathrm{AB} /(\mathrm{A} \times \mathrm{B})$. According to the absorbance of each group, $\mathrm{AB}$ is the ratio of the combination groups to control group; $\mathrm{A}$ or $\mathrm{B}$ is the ratio of the single agent group to control group. Thus, CDI value $<1,=1$ or $>1$ indicates that the drugs are synergistic, additive or antagonistic, respectively. MM, melanoma. 

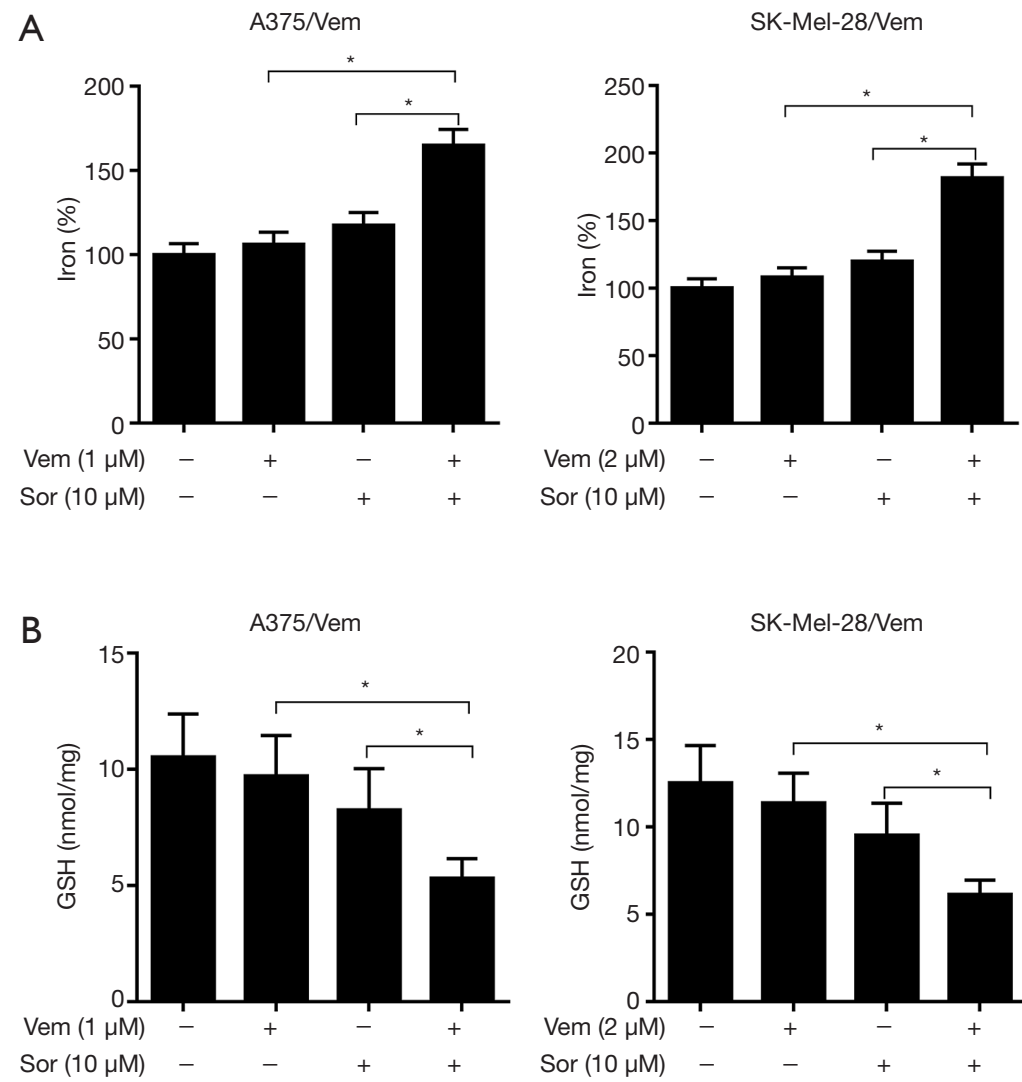

C

A375/Vem

SK-Mel-28/Vem
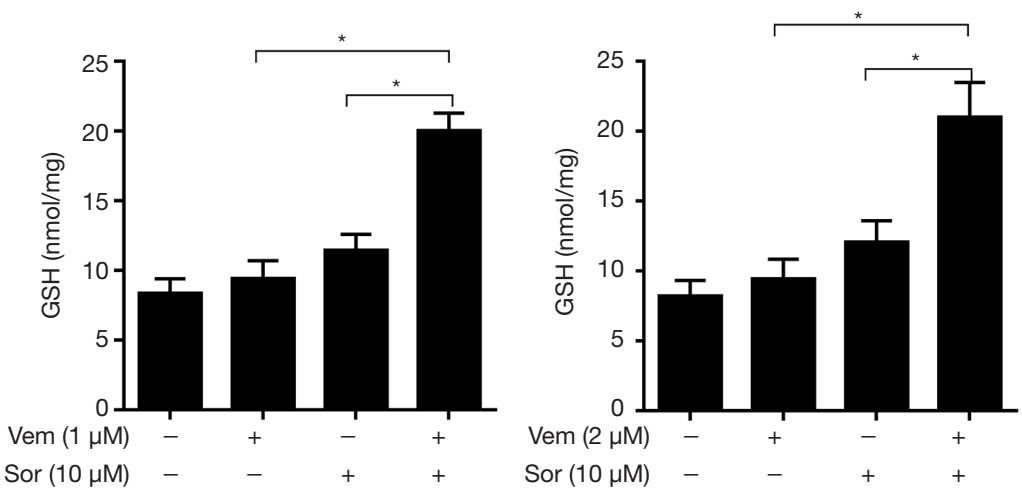

Figure 3 The effects of sorafenib on iron, GSH and MDA in vemurafenib-resistant MM cells. The cells were treated with vemurafenib or sorafenib alone, or both of them for 48 hours. (A) Iron Assay Kit was used to measure the iron concentration. (B) Glutathione Assay Kit was used to measure the GSH concentration. (C) Lipid Peroxidation (MDA) Assay Kit was used to measure the MDA concentration. * $\mathrm{P}<0.05$.

in cells treated with sorafenib alone or in combination with vemurafenib, and the effects induced by the drug combination were more intense than sorafenib treating alone (Figures S3,S4). These results implied that sorafenib may aggravated oxidative stress in vemurafenib resistant or treated melanoma cells.

\section{Sorafenib sensitizes melanoma cells to vemurafenib through ferroptosis}

In addition, we found that the combination of vemurafenib and sorafenib could suppress the antioxidant enzyme GPX4 and nuclear factor (erythroid-derived 2)-like 2 

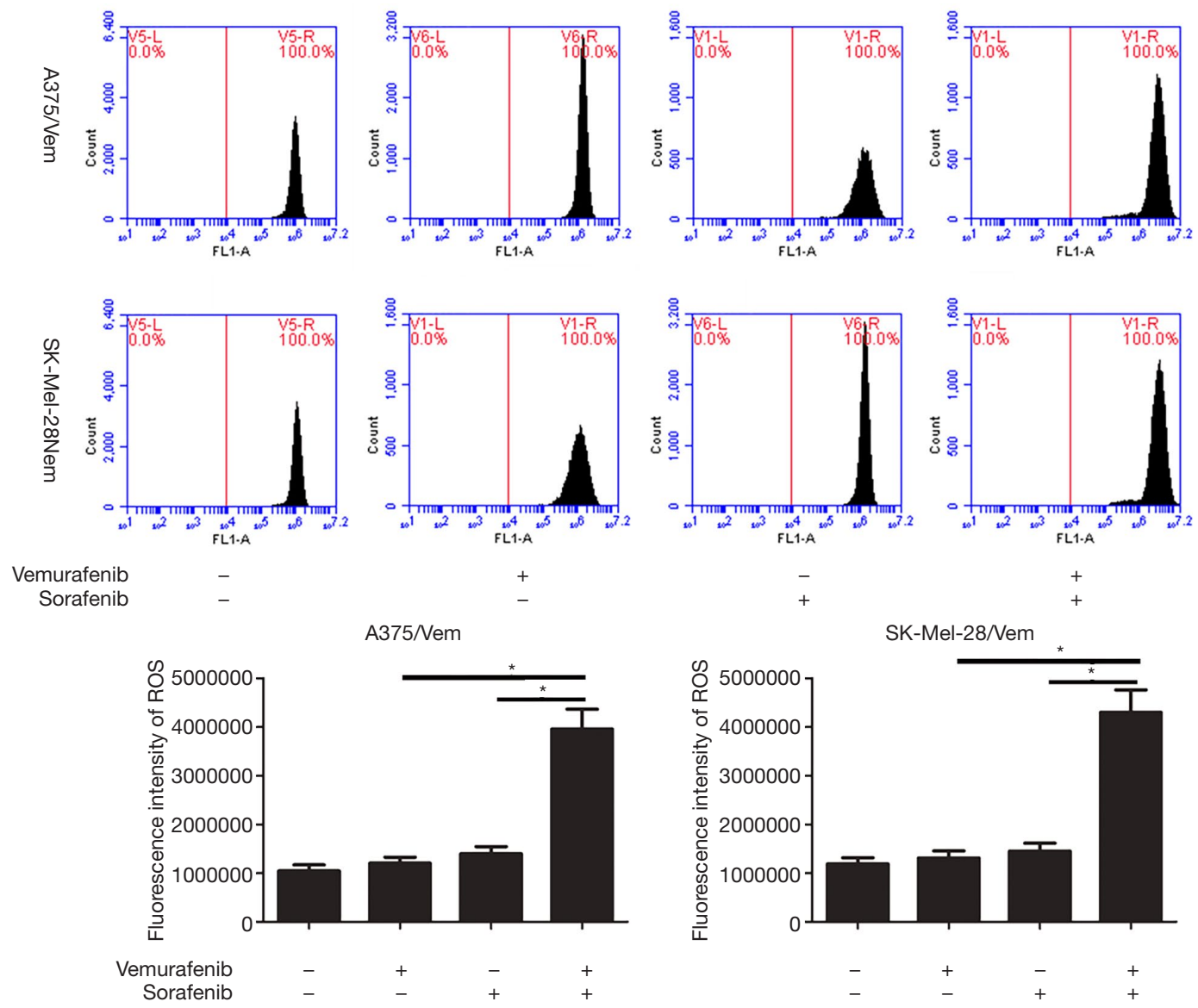

Figure 4 The effects of sorafenib on ROS production in vemurafenib-resistant MM cells. The cells were treated with vemurafenib or sorafenib alone, or both of them for 48 hours. Fluorometric Intracellular ROS Kit was used to measure the ROS concentration. * $\mathrm{P}<0.05$. ROS, reactive oxygen species; MM, melanoma.

(NRF2) (Figure 5). Vemurafenib resistant cells were treated with vemurafenib or sorafenib, and then exposed to the ferroptosis inhibitor Ferrostatin-1 (Fer-1), caspase inhibitor Z-VAD-FMK and MLKL inhibitor Necrosulfonamide. We found that the caspase inhibitor Z-VAD-FMK and MLKL inhibitor Necrosulfonamide did not significantly alter the inhibitory effects of vemurafenib and sorafenib (Figure 6). However, ferroptosis inhibitor Ferrostatin-1 treatment abolished the inhibitory effects of sorafenib or together with vemurafenib in A375/Vem and SK-Mel-28/Vem cells (Figure 6).

\section{Discussion}

MM is an aggressive disease. Molecular targeted therapies largely improved the overall survival of MM (20). BRAF inhibitors Vemurafenib is a drug that targeting to mutations in the BRAF/MEK/ERK pathway. The application of Vemurafenib results in a good clinical outcome as well as better safety and efficacy. However, BRAF inhibitors resistance brings out new targets in this pathway (21). One of the promising targets is the constitutive activation of the PI3K/AKT pathway that leading to intrinsic BRAF resistance (22). MAPK/ERK and PI3K/AKT pathways can be activated by c-Met (23), leading to drug resistance via upstream or downstream effectors (24).

In normal conditions, the MAPK signaling network was activated by receptor tyrosine kinases through RAS proteins. RAS can regulate BRAF in the MAPK pathway to trigger MEK 1/2 activation, and subsequent activation of ERK $1 / 2$ to induce several pro-growth factors and inhibit pro-apoptotic signals (25). A high percentage of melanomas have a mutated MEK1/2-activated MAPK signaling pathway (26), which leads to the promotion of 
A

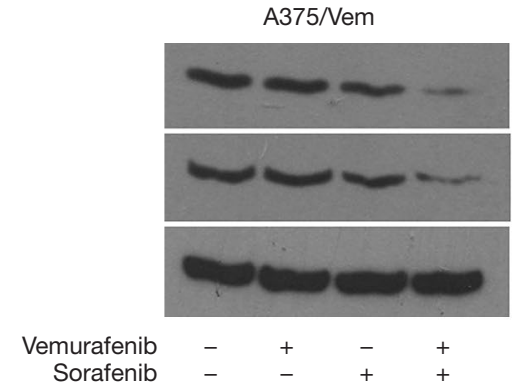

A375/Nem

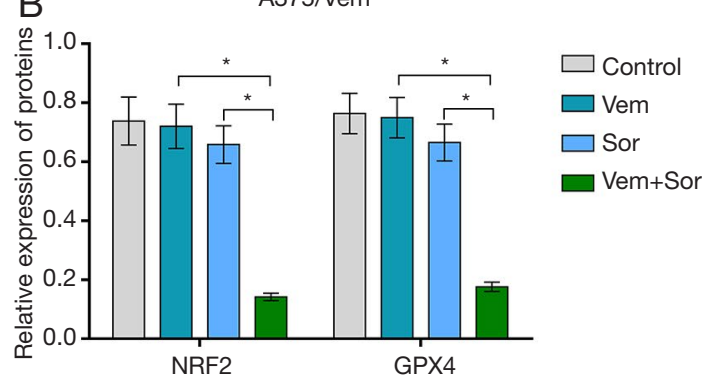

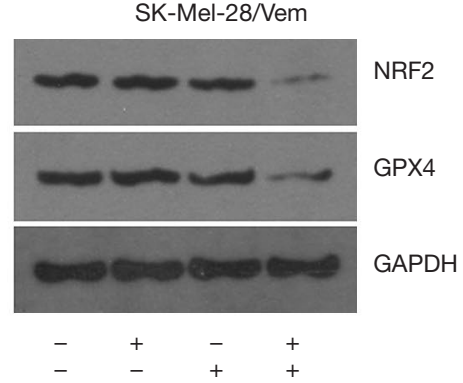

SK-Mel-28/Nem

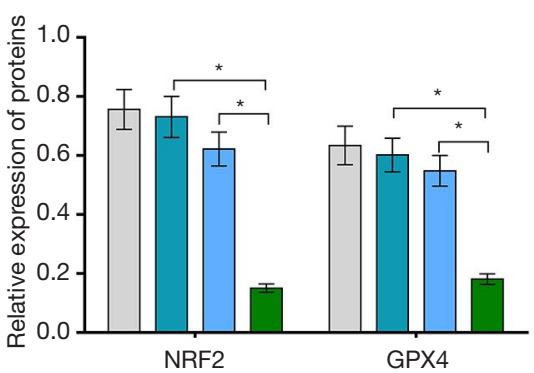

Figure 5 The effects of sorafenib on NRF2 and GPX4 in vemurafenib-resistant MM cells. The cells were treated with vemurafenib or sorafenib alone, or both of them for 48 hours. The untreated cells were used as control. (A) Western blot was performed to analyze the expression of NRF2 and GPX4. (B) The quantification of bands was shown in lower panel. *, $\mathrm{P}<0.05$. MM, melanoma.

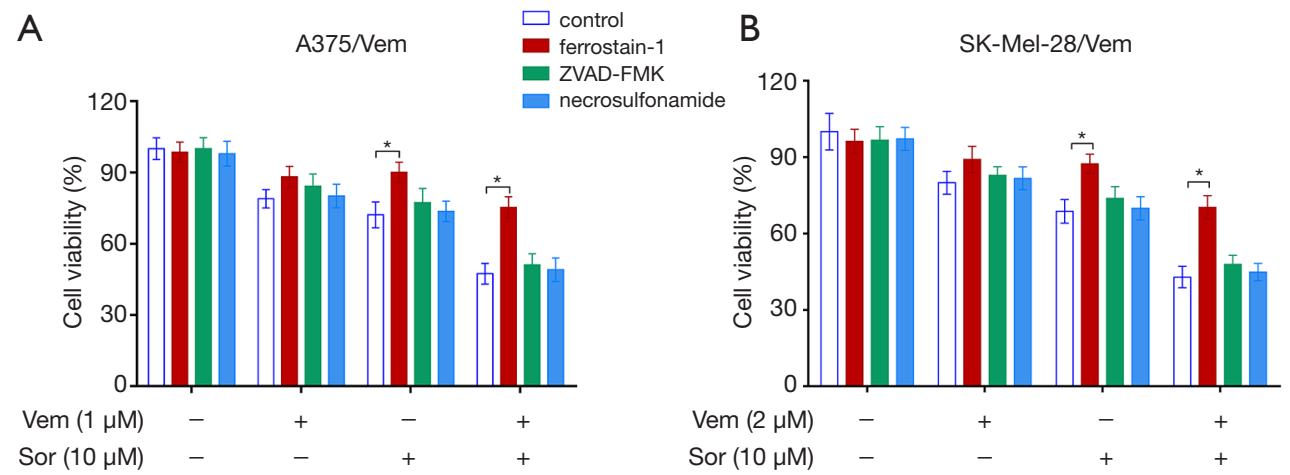

Figure 6 Lipid peroxidation inhibitors ferrostatin-1 reverses Sorafenib-mediated cell death. The cells were treated with vemurafenib or sorafenib alone, or both of them for 48 hours, and then exposed to ferroptosis inhibitor Ferrostatin-1 (Fer-1), caspase inhibitor Z-VAD-FMK and MLKL inhibitor Necrosulfonamide for 12 hours. CCK-8 assay was performed to measure the cell viability in vemurafenib-resistant A375 cells (A) and SK-Mel-28 cells (B). *, $\mathrm{P}<0.05$.

cell proliferation. However, these mutations also result in increased sensitivity to MAPK kinase inhibitors (20). Therefore, targeting MEK1/2 would be a promising candidate for cancer therapy (27). The addition of the MEK inhibitor cobimetinib to vemurafenib was associated with a significant improvement in progression-free survival among patients with BRAF V600-mutated metastatic melanoma (28).

Sorafenib inhibits tumor growth through disrupting tumor vasculature and targeting the MAPK pathway (29), including VEGFR, and platelet-derived growth factor receptor (PDGFR)- $\beta$ (1). Sorafenib can efficiently 
inhibited the growth of tumors that harboring k-ras or b-raf mutations in a dose-dependent manner, including melanoma (30). In addition, Sorafenib-induced death has also been shown to be associated with oxidative stress (31). Sorafenib could rapidly increase the production of ROS, inducing intracellular glutathione depletion in hepatocellular carcinoma (32). Sorafenib treatment resulted in ROS accumulation and necroptotic cell death activation in Hodgkin's lymphoma (33). It was also demonstrated that melatonin can increase the cytotoxic sensitivity of sorafenib through mitochondria stability and mitophagy in hepatocellular carcinoma cells (15). In this study, we found that sorafenib treatment did not significantly alter the production of ROS and the content of iron, GSH, and MDA in vemurafenib resistant melanoma cells, but cotreatment of sorafenib and vemurafenib dramatically upregulated ROS production, MDA and iron, but decreased GSH concentration. Interestingly, In WM 35 and A375 cells, the levels of Iron, GSH, MDA, and ROS were increased, but GSH were reduced in cells treated with sorafenib alone or in combination with vemurafenib. The indistunctive effects on oxidative stress by sorafenib in A375/Vem and SK-Mel-28/ Vem cells may be ascribe to the character of multidrug resistance, which contributed to a degree sorafenib resistance in A375/Vem and SK-Mel-28/Vem cells. Anyhow, these results implied that sorafenib may sensitize melanoma to vemurafenib through activation of oxidative stress. Moreover. sorafenib strongly promoted vemurafenib-mediated cell death. And the lipid peroxidation inhibitors ferrostatin-1 blocked these effects. Ferroptosis is characterized by the accumulation of iron-dependent ROS (34), and is can be induced sorafenib in cancer cells (35). MAPK activation and endoplasmic reticulum stress (ERS) upregulation are involved in ferroptosis (36). Ferroptosis is negatively regulated by glutathione peroxidase 4 (GPX4) and nuclear factor erythroid 2-related factor 2 (NRF2), which inhibits ROS accumulation and cellular iron uptake (37). Our recent results showed that cotreatment of sorafenib and vemurafenib suppressed GPX4 and NRF2 expression in vemurafenib resistant melanoma cells, which would increase ROS production and cellular iron uptake (38).

\section{Conclusions}

Our study identifies an association between sorafenib and ferroptosis in vemurafenib resistant melanoma cells. Our findings demonstrate that sorafenib treatment may be a novel strategy for overcoming vemurafenib resistance in melanoma.

\section{Acknowledgments}

Funding: This work was supported by the New Xiangya Talent Project of the Third Xiangya Hospital of Central South University (JY201607).

\section{Footnote}

Conflicts of Interest: All authors have completed the ICMJE uniform disclosure form (available at http://dx.doi. org/10.21037/tcr.2020.01.62). The authors have no conflicts of interest to declare.

Ethical Statement: The authors are accountable for all aspects of the work in ensuring that questions related to the accuracy or integrity of any part of the work are appropriately investigated and resolved. The study was conducted in accordance with the Declaration of Helsinki (as revised in 2013). This institutional ethical approval and informed consent were waived.

Open Access Statement: This is an Open Access article distributed in accordance with the Creative Commons Attribution-NonCommercial-NoDerivs 4.0 International License (CC BY-NC-ND 4.0), which permits the noncommercial replication and distribution of the article with the strict proviso that no changes or edits are made and the original work is properly cited (including links to both the formal publication through the relevant DOI and the license). See: https://creativecommons.org/licenses/by-nc-nd/4.0/.

\section{References}

1. Siegel RL, Miller KD, Jemal A. Cancer statistics, 2018. CA Cancer J Clin 2018;68:7-30.

2. Zhou J, Xu D, Xie H, et al. miR-33a functions as a tumor suppressor in melanoma by targeting HIF-1alpha. Cancer Biol Ther 2015;16:846-55.

3. Zhou J, Liu R, Luo C, et al. MiR-20a inhibits cutaneous squamous cell carcinoma metastasis and proliferation by directly targeting LIMK1. Cancer Biol Ther 2014;15:1340-49.

4. Liu C, He SQ, Chen XQ, et al. Research Advances in the Treatment of Melanoma by Treat Melanoma. Curr Top Med Chem 2015;16:242-50.

5. Corazao-Rozas P, Guerreschi P, Jendoubi M, et al. Mitochondrial oxidative stress is the Achille's heel of melanoma cells resistant to Braf-mutant inhibitor. Oncotarget 2013;4:1986-98. 
6. Johannessen CM, Boehm JS, Kim SY, et al. COT drives resistance to RAF inhibition through MAP kinase pathway reactivation. Nature 2010;468:968-72.

7. Kim A, Cohen MS. The discovery of vemurafenib for the treatment of BRAF-mutated metastatic melanoma. Expert Opin Drug Discov 2016;11:907-16.

8. Yang WS, SriRamaratnam R, Welsch ME, et al. Regulation of ferroptotic cancer cell death by GPX4. Cell 2014;156:317-31.

9. Luo $\mathrm{M}, \mathrm{Wu} \mathrm{L}$, Zhang $\mathrm{K}$, et al. miR-137 regulates ferroptosis by targeting glutamine transporter SLC1A5 in melanoma. Cell Death Differ 2018;25:1457-72.

10. Bai T, Wang S, Zhao Y, et al. Haloperidol, a sigma receptor 1 antagonist, promotes ferroptosis in hepatocellular carcinoma cells. Biochem Biophys Res Commun 2017;491:919-25.

11. Yang WS, Stockwell BR. Synthetic lethal screening identifies compounds activating iron-dependent, nonapoptotic cell death in oncogenic-RAS-harboring cancer cells. Chem Biol 2008;15:234-45.

12. Yagoda N, von Rechenberg M, Zaganjor E, et al. RAS-RAF-MEK-dependent oxidative cell death involving voltage-dependent anion channels. Nature 2007;447:864-8.

13. Severi T, van Malenstein H, Verslype C, et al. Tumor initiation and progression in hepatocellular carcinoma: risk factors, classification, and therapeutic targets. Acta Pharmacol Sin 2010;31:1409-20.

14. Nguyen K, Jack K, Sun W. Hepatocellular Carcinoma: Past and Future of Molecular Target Therapy. Diseases 2015. doi: 10.3390/diseases4010001.

15. Prieto-Domínguez N, Ordonez R, Fernandez A, et al. Melatonin-induced increase in sensitivity of human hepatocellular carcinoma cells to sorafenib is associated with reactive oxygen species production and mitophagy. $\mathrm{J}$ Pineal Res 2016;61:396-407.

16. Lange M, Abhari BA, Hinrichs TM, et al. Identification of a novel oxidative stress induced cell death by Sorafenib and oleanolic acid in human hepatocellular carcinoma cells. Biochem Pharmacol 2016;118:9-17.

17. Marra M, Sordelli IM, Lombardi A, et al. Molecular targets and oxidative stress biomarkers in hepatocellular carcinoma: an overview. J Transl Med 2011;9:171.

18. Tsoi J, Robert L, Paraiso K, et al. Multi-stage Differentiation Defines Melanoma Subtypes with Differential Vulnerability to Drug-Induced Iron-Dependent Oxidative Stress. Cancer Cell 2018;33:890-904.e5.

19. Mologni L, Costanza M, Sharma GG, et al. Concomitant
BCORL1 and BRAF Mutations in Vemurafenib-Resistant Melanoma Cells. Neoplasia 2018;20:467-77.

20. Faghfuri E, Nikfar S, Niaz K, et al. Mitogen-activated protein kinase (MEK) inhibitors to treat melanoma alone or in combination with other kinase inhibitors. Expert Opin Drug Metab Toxicol 2018;14:317-30.

21. Boada A, Carrera C, Segura S, et al. Cutaneous toxicities of new treatments for melanoma. Clin Transl Oncol 2018;20:1373-84.

22. Wahid M, Jawed A, Mandal RK, et al. Recent developments and obstacles in the treatment of melanoma with BRAF and MEK inhibitors. Crit Rev Oncol Hematol 2018;125:84-8.

23. Marchetti P, Trinh A, Khamari R, et al. Melanoma metabolism contributes to the cellular responses to MAPK/ERK pathway inhibitors. Biochim Biophys Acta Gen Subj 2018;1862:999-1005.

24. Lim SY, Menzies AM, Rizos H. Mechanisms and strategies to overcome resistance to molecularly targeted therapy for melanoma. Cancer 2017;123:2118-29.

25. Qiao A, Wang K, Yuan Y, et al. Sirt3-mediated mitophagy protects tumor cells against apoptosis under hypoxia. Oncotarget 2016;7:43390-400.

26. Xie B, Cao K, Li J, et al. Hmgb1 inhibits Klotho expression and malignant phenotype in melanoma cells by activating NF-kappaB. Oncotarget 2016;7:80765-82.

27. Hepner A, Salgues A, Anjos C, et al. Treatment of advanced melanoma - A changing landscape. Rev Assoc Med Bras (1992) 2017;63:814-23.

28. Larkin J, Ascierto PA, Dreno B, et al. Combined vemurafenib and cobimetinib in BRAF-mutated melanoma. N Engl J Med 2014;371:1867-76.

29. Strumberg D, Clark JW, Awada A, et al. Safety, pharmacokinetics, and preliminary antitumor activity of sorafenib: a review of four phase I trials in patients with advanced refractory solid tumors. Oncologist 2007;12:426-37.

30. Ryu S, Youn C, Moon AR, et al. Therapeutic Inhibitors against Mutated BRAF and MEK for the Treatment of Metastatic Melanoma. Chonnam Med J 2017;53:173-7.

31. Shen YC, Ou DL, Hsu C, et al. Activating oxidative phosphorylation by a pyruvate dehydrogenase kinase inhibitor overcomes sorafenib resistance of hepatocellular carcinoma. Br J Cancer 2013;108:72-81.

32. Chiou JF, Tai CJ, Wang YH, et al. Sorafenib induces preferential apoptotic killing of a drug- and radio-resistant Hep G2 cells through a mitochondria-dependent oxidative stress mechanism. Cancer Biol Ther 2009;8:1904-13. 
33. Locatelli SL, Cleris L, Stirparo GG, et al. BIM upregulation and ROS-dependent necroptosis mediate the antitumor effects of the HDACi Givinostat and Sorafenib in Hodgkin lymphoma cell line xenografts. Leukemia 2014;28:1861-71.

34. Louandre C, Marcq I, Bouhlal H, et al. The retinoblastoma $(\mathrm{Rb})$ protein regulates ferroptosis induced by sorafenib in human hepatocellular carcinoma cells. Cancer Lett 2015;356:971-7.

35. Lachaier E, Louandre C, Godin C, et al. Sorafenib induces ferroptosis in human cancer cell lines originating from

Cite this article as: Tang F, Li S, Liu D, Chen J, Han C. Sorafenib sensitizes melanoma cells to vemurafenib through ferroptosis. Transl Cancer Res 2020;9(3):1584-1593. doi: $10.21037 /$ tcr.2020.01.62 different solid tumors. Anticancer Res 2014;34:6417-22.

36. Sun X, Ou Z, Chen R, et al. Activation of the p62-Keap1NRF2 pathway protects against ferroptosis in hepatocellular carcinoma cells. Hepatology 2016;63:173-84.

37. Xie Y, Hou W, Song X, et al. Ferroptosis: process and function. Cell Death Differ 2016;23:369-79.

38. Li PC, Tu MJ, Ho PY, et al. Bioengineered NRF2-siRNA Is Effective to Interfere with NRF2 Pathways and Improve Chemosensitivity of Human Cancer Cells. Drug Metab Dispos 2018;46:2-10. 


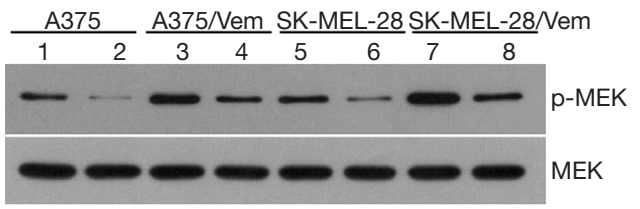

Figure S1 The vemurafenib-resistant cells maintained a highly level of phospho-MEK in the presence of vemurafenib. 1, 3, 5, 7: respectively indicate each cell culturing without vemurafenib; 2, 4, 6, 8: respectively indicate each cell culturing in the presence of vemurafenib, and the drug concentrations were $0.2,1,0.4,2 \mu \mathrm{M}$, respectively.
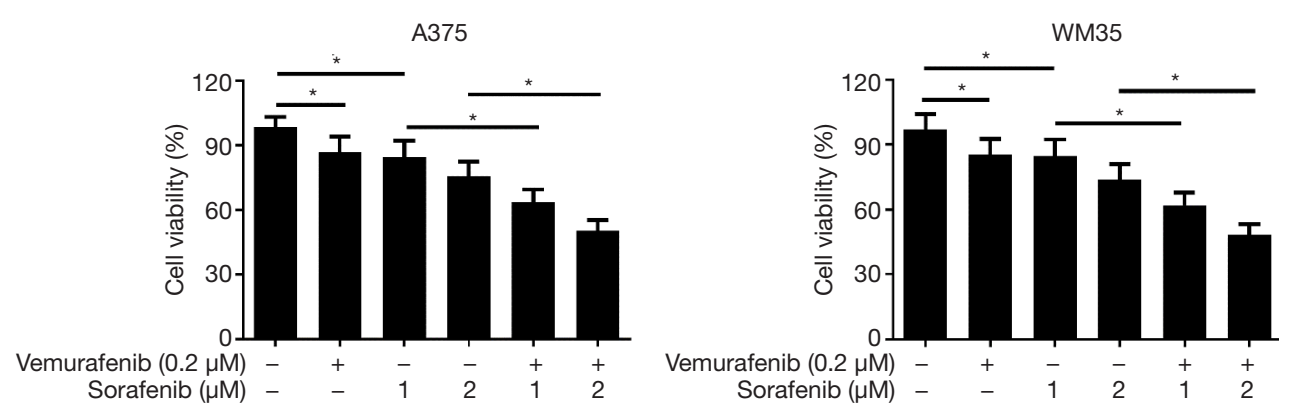

Figure S2 Sorafenib sensitizes A375 and WM35 cells to vemurafenib. The A375 and WM35 cells were treated with vemurafenib or sorafenib alone, or both of them for 48 hours. CCK-8 assay was performed to measure the cell viability after 48 h-treatment. *, $\mathrm{P}<0.05$. 
A

A375
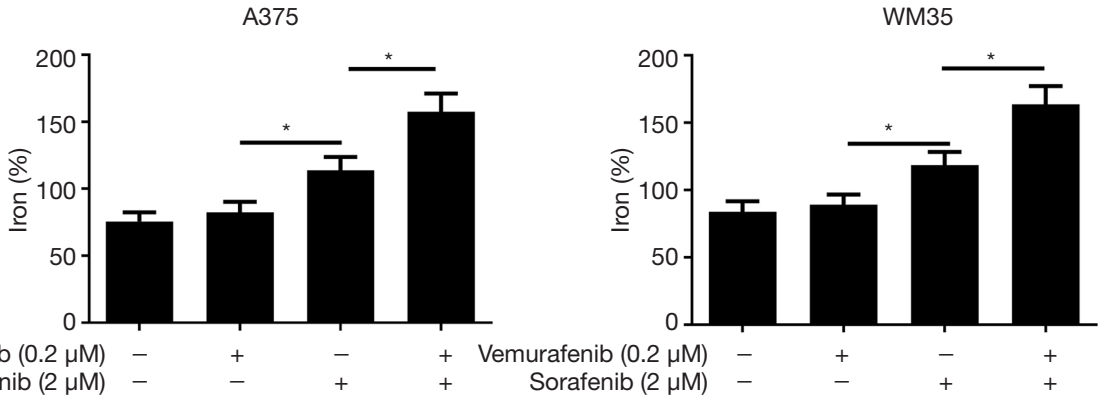

B

A375

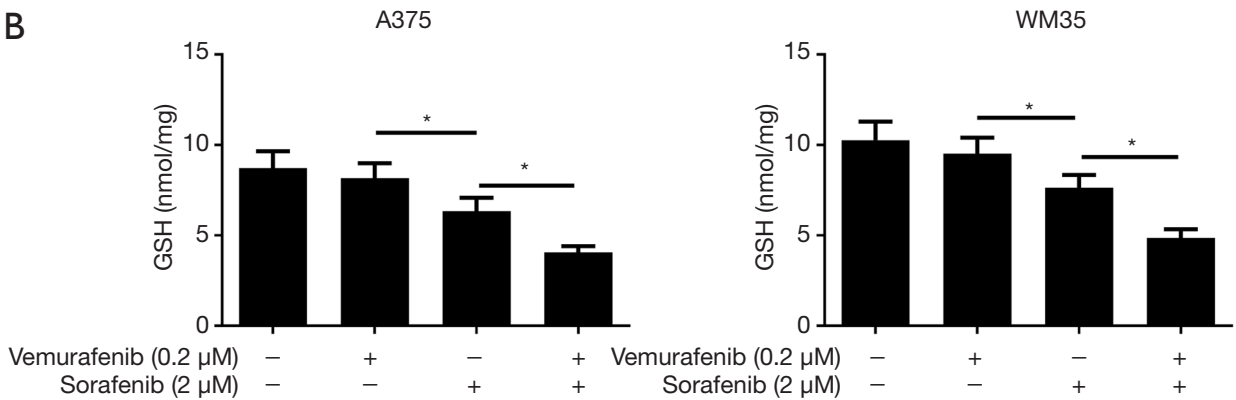

WM35

C

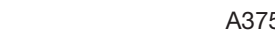

A375

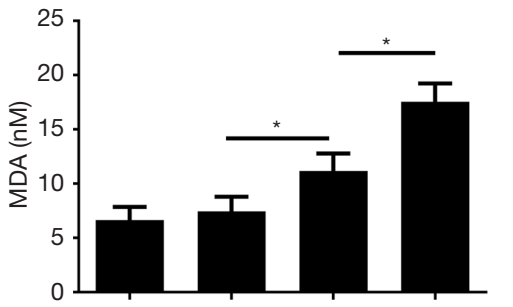

Vemurafenib $(0.2 \mu \mathrm{M})$

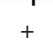

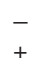

$+$

emurafenib $(0.2 \mu \mathrm{M})$

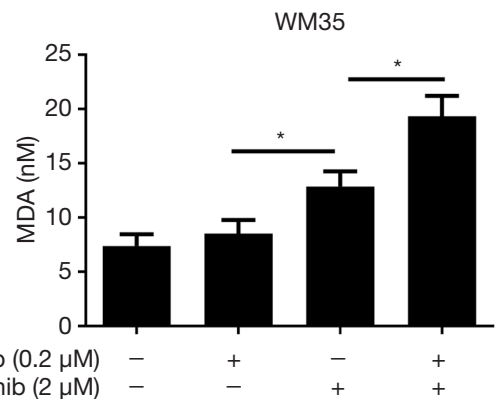

Figure S3 The effects of sorafenib on iron, GSH and MDA in A375 and WM35 cells. The cells were treated with Vemurafenib or sorafenib alone, or both of them for 48 hours. (A) Iron Assay Kit was used to measure the iron concentration. (B) Glutathione Assay Kit was used to measure the GSH concentration. (C) Lipid Peroxidation (MDA) Assay Kit was used to measure the MDA concentration. *, $\mathrm{P}<0.05$. 

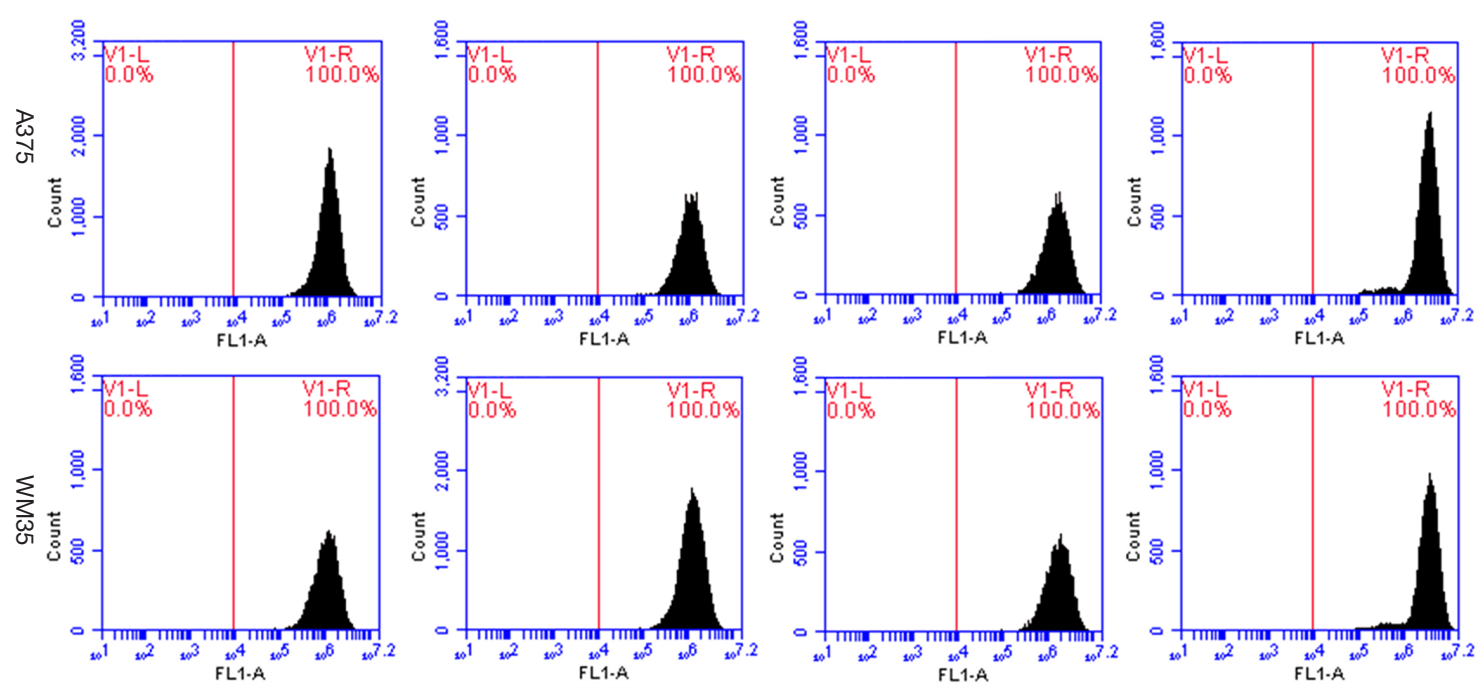

Vemurafenib

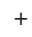

Sorafenib

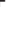

A375
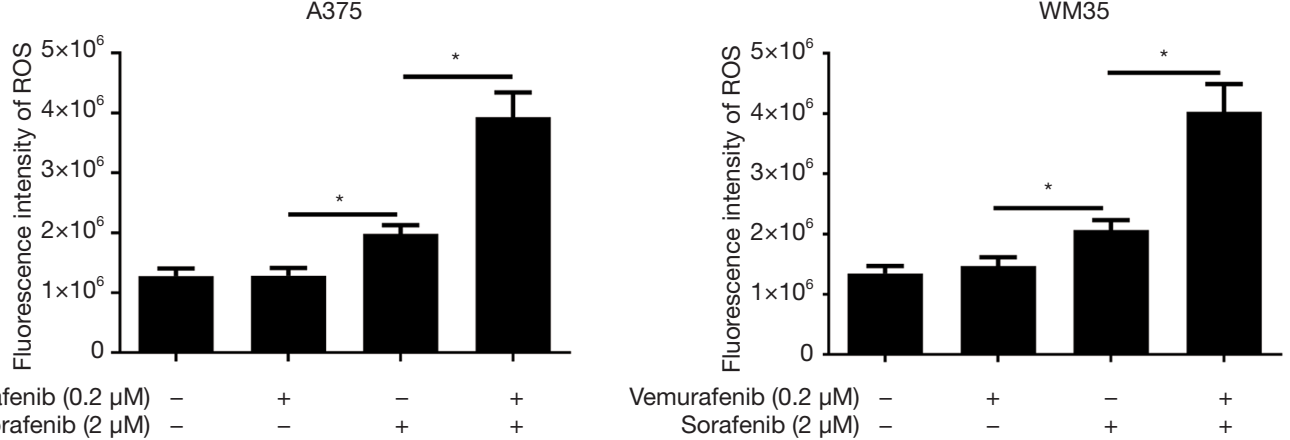

Figure S4 The effects of sorafenib on ROS production in A375 and WM35 cells. The cells were treated with vemurafenib or sorafenib alone, or both of them for 48 hours. Fluorometric Intracellular ROS Kit was used to measure the ROS concentration. *, P<0.05. ROS, reactive oxygen species. 